\title{
Growth Differentiation Factor-15 in Immunity and Aging
}

\author{
Brandt D. Pence* \\ College of Health Sciences, University of Memphis, Memphis, TN, United States
}

Aging increases susceptibility to and severity of a variety of chronic and infectious diseases. Underlying this is dysfunction of the immune system, including chronic increases in lowgrade inflammation (inflammaging) and age-related immunosuppression (immunosenescence). Growth differentiation factor-15 (GDF-15) is a stress-, infection-, and inflammation-induced cytokine which is increased in aging and suppresses immune responses. This mini review briefly covers existing knowledge on the immunoregulatory and anti-inflammatory roles of GDF-15, as well as its potential importance in aging and immune function.

Keywords: inflammaging, immunosenescence, aging, immunity, GDF-15

\section{INTRODUCTION}

OPEN ACCESS

Edited by:

Leena P. Bharath,

Merrimack College, United States

Reviewed by:

Jessica Naomi Lancaster, Mayo Clinic Arizona, United States

Jan Nehlin,

Copenhagen University Hospital Amager and Hvidovre, Denmark

${ }^{*}$ Correspondence:

Brandt D. Pence

bdpence@memphis.edu

Specialty section: This article was submitted to Aging and the Immune System, a section of the journal

Frontiers in Aging

Received: 16 December 2021

Accepted: 24 January 2022

Published: 09 February 2022

Citation:

Pence BD (2022) Growth Differentiation Factor-15 in Immunity and Aging.

Front. Aging 3:837575. doi: 10.3389/fragi.2022.837575
Aging is the single largest risk factor for nearly all chronic diseases, including cardiovascular disease, cancer, and neurodegenerative diseases (Niccoli and Partridge, 2012). Underlying nearly all chronic diseases is an increase in inflammation, and numerous observations have associated aging with a chronic low-grade inflammatory state. This has given rise to the term inflammaging, in which ageassociated inflammation is suggested to be a shared underlying cause for the progressive decline in physiological function and increased pathology with age (Franceschi and Campisi, 2014). It is now well-appreciated that dysregulated inflammation is closely coupled with the aging phenotype, as inflammatory processes are central to essentially all of the 'hallmarks of aging' defined in a landmark paper (Lopez-Otin et al., 2013) published in Cell in 2013. Systemic chronic inflammation underlies age-related disease processes across tissue types (Furman et al., 2019) and is predictive of multimorbidity and frailty (Sayed et al., 2021), suggesting that inflammaging is central to biological aging.

In addition to the increase in inflammation seen in the inflammaging state, the aging process also brings about progressive immunosenescence, a generalized decline in immune system function leading to increased complications from infectious diseases and other immunological stimuli (Fulop et al., 2018). Immunosenescence leads to defects in the innate immune system, including impaired phagocytosis and chemotaxis, increased myeloid cell proportion, and altered basal and stimulated cytokine production in granulocytes, monocytes, macrophages, and dendritic cells (Linton and Thoman, 2014; Fulop et al., 2018). Additionally, adaptive immune responses are generally impaired with aging, as lymphocyte subpopulations shift to more regulatory and memory phenotypes, and lymphocyte proliferation and function is decreased (Goronzy and Weyand, 2013; Fulop et al., 2018). Because inflammatory responses are a major aspect of immune regulation, inflammaging and immunosenescence are invariably linked.

However, some aspects of aging and immunity are paradoxical (Montgomery and Shaw, 2015), as anti-inflammatory and immunoregulatory cell subtypes are proportionally increased during the aging process (Fulop et al., 2018), which would be expected to promote a more anti-inflammatory state. Additionally, immune cells are known to take on more pro- or anti-inflammatory roles during 
aging depending on stimulus, as exemplified by research on monocytes showing age-related increased basal expression of the pro-inflammatory cytokine tumor necrosis factor (TNF)- $\alpha$ (Hearps et al., 2012), while cytokine responses are impaired with aging during inflammatory activation in monocytes (Renshaw et al., 2002; Pence and Yarbro, 2019).

\section{SENESCENCE}

A principal contributor to the aging process is cellular senescence (Lopez-Otin et al., 2013; Yarbro et al., 2020). One of the 'hallmarks of aging' (Lopez-Otin et al., 2013), cellular senescence is characterized by proliferative arrest in aging cells, preventing cell division in an irreversible manner (Campisi, 2012). However, despite the similarity in their names, cellular senescence and immunosenescence are different processes with widely-varying outcomes. Immunosenescence refers to a generalized deterioration in immune cell function during aging, predisposing older individuals to worsened outcomes to infectious and chronic diseases (Fulop et al., 2018). While there is some evidence for cellular senescence in the immune system, primarily in lymphocytes (Zhou et al., 2021), mechanisms underlying immunosenescence are not universally driven by hallmarks of cellular senescence such as cell cycle arrest, telomere shortening, etc. Nevertheless, links between cellular senescence and immunosenescence are a promising area of research, as there has been a dramatic increase in interest in the regulation of host processes by senescent cells, driven primarily by the discovery of the senescence-associated secretory phenotype (SASP).

During aging, senescent cells produce a host of secreted factors now known as the SASP, which are involved in the regulation of myriad host functions including immune system-relevant processes such as inflammation, tissue repair, and cellular proliferation (Campisi, 2012). Many SASP factors are cytokines and chemokines which are intimately involved in the regulation of inflammation (Campisi et al., 2011), thus cellular senescence is a primary driver of inflammaging both at local tissue and systemic levels (Freund et al., 2010) and contributes to the pro-inflammatory environment further induced by other age-associated factors such as increases in damage-associated molecular patterns (Kapetanovic et al., 2015). As a major determinant of the host endocrine environment, SASP factors are also prime candidates for potential mechanisms linking cellular senescence and inflammaging to immunosenescence, as many circulating and tissue immune cells are routinely exposed to secreted SASP factors.

\section{GDF-15}

Growth Differentiation Factor-15 (GDF-15) is a distant member of the transforming growth factor (TGF)- $\beta$ superfamily of cytokines (Unsicker et al., 2013), in that it shares structural characteristics with TGF- $\beta$ superfamily members but was found to have relatively weak homology with existing superfamily members at the time of its discovery (Bootcov et al., 1997). GDF-15 has detectable expression in nearly all tissues including the brain, intestines, lungs, cardiovascular system, etc. (Yokoyama-Kobayashi et al., 1997; Böttner et al., 1999; Tan et al., 2002).

GDFs have a long history in aging research (Jamaiyar et al., 2017), although this has been controversial due to debates about the direction and effects of age-associated changes to key GDFs including myostatin (GDF-8) and GDF-11 (Loffredo et al., 2013; Katsimpardi et al., 2014; Sinha et al., 2014; Egerman et al., 2015; Smith et al., 2015; Poggioli et al., 2016). However, the known effects of GDF-15 are distinct from those of GDF-8, GDF-11, and other proteins of this subfamily relevant to aging (Unsicker et al., 2013), and GDF-15 is sufficiently divergent from other TGF- $\beta$ superfamily members that it was initially suggested to be the first member of a new subfamily of TGF- $\beta$-related proteins (Bootcov et al., 1997).

GDF-15 was independently discovered by multiple laboratories in the late 1990s (Bootcov et al., 1997; Hromas et al., 1997; Lawton et al., 1997; Baek et al., 2001), with each laboratory describing a distinct function of the protein. The most influential of these initial publications (by citation count) was from Bootcov et al. in 1997 (Bootcov et al., 1997), who named GDF-15 as macrophage inhibitory cytokine-1 (MIC-1) and demonstrated 1) that GDF-15 was released by macrophages due to inflammatory stimuli such as tumor necrosis factor (TNF)- $\alpha$ and interleukin (IL)-1 $\beta$, and 2) that GDF-15 signaling in macrophages inhibits lipopolysaccharidestimulated TNF-a production. This seminal manuscript provided evidence that GDF-15 is an important immunoregulatory protein which links an inflammatory state with immunosuppression. Follow-up work from several laboratories has implicated GDF-15 in suppressing function of a variety of immune cells, including neutrophils (Kempf et al., 2011; Artz et al., 2016; Zhang et al., 2016), macrophages (Preusch et al., 2013; Lee et al., 2017; Jung et al., 2018), dendritic cells (Segerer et al., 2012; Zhou et al., 2013; Zhang et al., 2018), natural killer (NK) cells (Roth et al., 2010; Kleinertz et al., 2019), and T lymphocytes (Roth et al., 2010).

In addition to its molecular effects, GDF-15 is well-established as a biomarker for a number of chronic diseases, many of which are increased with age. Increased GDF-15 levels have been associated with cardiovascular disease (Wollert et al., 2017), mitochondrial diseases (Yatsuga et al., 2015), diabetes (Adela and Banerjee, 2015), and cognitive decline (Fuchs et al., 2013) among others. GDF-15 is also an active area of investigation within cancer research, as it has dually-opposing effects including both anti-tumorigenic and pro-metastatic activities depending on cell type studied (Unsicker et al., 2013). Increased GDF-15 has also been suggested as a biomarker for severity of rheumatoid arthritis (Esalatmanesh et al., 2020).

The independent identification by multiple laboratories of GFRAL as the neuronal receptor for GDF-15 (Emmerson et al., 2017; Mullican et al., 2017; Yang et al., 2017) which mediates its known anti-obesity effects has also accelerated research into GDF-15 as a weight-loss promoter. However, the 
long-term consequences of increasing GDF-15 are questionable, given its association with immunosuppression and various chronic diseases. It is also worth noting that GDF-15 signaling mechanisms are likely to be distinct in different cell types, as canonical TGF- $\beta$ receptor signaling has been shown to mediate the effects of GDF-15 in various immune cells (Artz et al., 2016; Jung et al., 2018; Zhang et al., 2018; Kleinertz et al., 2019). Importantly, immune cells to do not express GFRAL, necessitating that the observed effects of GDF-15 on leukocytes occur through an alternate receptor.

Further underscoring GFRAL-independent aspects of GDF-15 signaling is the recent observation that GDF-15 activates AMPK in skeletal muscle independent of GFRAL (Aguilar-Recarte et al., 2021). The geroprotector drug metformin also increases circulating GDF-15 levels and promotes weight loss, and GDF15 knockout abrogates the weight loss effect of metformin in mice (Day et al., 2019). While the GDF-15 mediated weight reduction effects of metformin may be mediated through GFRAL, metformin is also well known as an anti-inflammatory and immunomodulatory drug (Bułdak et al., 2014; Kim et al., 2014; Qing et al., 2019; Soberanes et al., 2019; Cory et al., 2021; Xian et al., 2021), therefore the effect of metformin on inflammation and immune function may be at least partially mediated through promoting GDF-15 expression.

\section{GDF-15 AND IMMUNITY}

In addition to suppressing inflammatory responses (Roth et al., 2010; Kempf et al., 2011; Segerer et al., 2012; Preusch et al., 2013; Zhou et al., 2013; Artz et al., 2016; Zhang et al., 2016; Lee et al., 2017; Jung et al., 2018; Zhang et al., 2018), GDF-15 is a potent suppressor of chemotaxis in neutrophils (Kempf et al., 2011; Artz et al., 2016; Zhang et al., 2016), restricts macrophage accumulation in atherosclerotic plaques (Preusch et al., 2013), and promotes autophagy in macrophages (Heduschke et al., 2021). In macrophages, GDF-15 increases reliance on oxidative phosphorylation for energy production and promotes an M2-like phenotype (Jung et al., 2018), which suggests a potential mechanism by which GDF-15 mediates antiinflammatory responses. GDF-15 also reduces LPS-induced sepsis responses in mice (Abulizi et al., 2017) and suppresses NLRP3 inflammasome activation and inflammatory responses in adipose tissue (Kim et al., 2013; Wang et al., 2014). These findings suggest that GDF-15 is a potent suppressor of inflammatory responses by innate immune cells.

Some evidence suggests that GDF-15 can suppress DC function. In perhaps the most comprehensive study to date, Zhou et al. (Zhou et al., 2013) demonstrated impaired expression of maturation markers, reduced inflammatory cytokine production, and impaired $\mathrm{T}$ cell activation by GDF15-treated DCs. These findings are consistent with a study on decidual dendritic cells, which demonstrated impaired maturation and $\mathrm{T}$ cell stimulatory capacity in GDF-15-treated DCs (Segerer et al., 2012). GDF-15 has also been shown to promote tolerogenic DC responses, including increasing expression of inhibitory molecules and promoting $\mathrm{T}$ cell exhaustion and regulatory $\mathrm{T}$ cell production by DCs (Zhang et al., 2018). Aging is known to impair DC function, including by reducing antigen presentation (Wong and Goldstein, 2013). Agerelated increases in GDF-15 therefore represents a potential mechanism for the observation of DC impairments during aging.

GDF-15 is also an active area of study in cancer immunity (Wischhusen et al., 2020; Lodi et al., 2021), and GDF-15 has been shown to allow gliomas to evade immune responses by suppressing natural killer cell-mediated immunity and $\mathrm{T}$ cell migration into the tumors (Roth et al., 2010). GDF-15 also suppresses macrophage anti-tumor responses during early cancer development (Ratnam et al., 2017). GDF-15 is highly overexpressed in colorectal, ovarian, lung, and many other cancer types (Wischhusen et al., 2020), and is therefore considered a promising biomarker for cancer prognosis in addition to its potential role in cancer cell evasion of anti-tumor immune responses.

GDF-15 is additionally relevant to infectious disease responses. GDF-15 impairs NK cell function during systemic infection (Kleinertz et al., 2019) and regulates polarization of adipose tissue macrophages (Lee et al., 2017). One recent paper demonstrated that GDF-15 overproduction increases severity of human rhinovirus infections (Wu et al., 2017), and Kleinertz et al. reported that GDF-15 levels were increased in injury patients who progressed to sepsis compared to those who did not (Kleinertz et al., 2019). Likewise, GDF-15 expression is increased in cells infected with avian influenza viruses, and this in turn limits their production of cytokines (Zhao et al., 2021). COVID-19 patients have elevated GDF-15 levels (Notz et al., 2020; Rochette et al., 2021), suggesting a potential link between GDF-15 and disease severity in the ongoing pandemic.

In septic patients, GDF-15 levels are predictive of disease severity and mortality (Buendgens et al., 2017), giving further evidence that GDF-15 may be of import in systemic immune responses. Finally, GDF-15 has been shown to be released due to infection with a variety of bacterial and viral pathogens, and that it plays a tissue protective role during infection via regulation of lipid metabolism (Luan et al., 2019). As such, there is abundant evidence that GDF-15 plays a multifaceted role in immune responses by down-regulating immune function, not unlike impairments to the immune system noted in age-related immunosenescence.

\section{GDF-15 AND AGING}

Within the aging field, GDF-15 has very recently become a protein of tremendous interest. Although GDF-15 was identified in 2010 as being associated with all-cause mortality in Swedish males (Wiklund et al., 2010), the protein was largely forgotten in the aging field until a 2018 publication in Aging Cell by Tanaka et al. (Tanaka et al., 2018) profiled the plasma proteome across the lifespan. They demonstrated that GDF-15 was the protein most strongly associated with age and that it increased in a linear fashion as age increased. Follow-up studies by the same authors indicated that GDF-15 was the protein most strongly 
associated with multimorbidity, including after adjustment for age and sex (Tanaka et al., 2020).

At least several additional papers support the finding that GDF-15 levels increase with age (Ho et al., 2012; Doerstling et al., 2018), and elevated GDF-15 levels are associated with the development of anemia in older adults (Yamaguchi et al., 2021). Underscoring this, Tavenier et al. recently described a strong association between GDF-15 levels and accelerated aging phenotypes in older adults, wherein individuals with frailty had on average an approximately $60 \%$ increase in GDF-15 compared to age-matched healthy individuals (Tavenier et al., 2021). This finding supported a previous report linking GDF-15 levels in the plasma to frailty (Conte et al., 2020). Although in need of further support, these findings suggest GDF-15 as potentially prognostic of biological aging.

A recent study by Basisty et al. (Basisty et al., 2020) profiled the SASP across multiple cell types and in vitro senescence inducers with the goal of developing a "SASP Atlas" to support research in this field. GDF-15 was identified as part of the "core SASP" which was upregulated across cell types and treatments, confirming its importance as potential signaling molecule in cellular senescence. These recent findings underscore the potential importance of GDF-15 to aging, although the actual molecular contributions of GDF-15 to aging are currently unknown.

Given its relatively recent emergence as a biomarker of aging, it is unsurprising that little is known about the contribution of GDF-15 to immunosenescence. My laboratory has recently described correlations between elevated GDF-15 levels and monocyte dysfunction (Pence et al., 2021) in a secondary analysis using data from our previous reports on age-related metabolic and inflammatory deficits in monocytes (Pence and Yarbro, 2018; Pence and Yarbro, 2019). Interestingly, Moon et al. (Moon et al., 2020) recently reported that GDF-15 is increased in aging in response to cell-free mitochondrial DNA, and that this limits tissue inflammatory burden. However, GDF-15 also suppressed T cell activation via promoting regulatory $\mathrm{T}$ cell activity, thereby limiting immune activation. Circulating GDF-15 levels were additionally recently shown to be correlated to accumulation of senescent $\mathrm{T}$ cells during aging (Chen et al., 2020). GDF-15 may therefore be a regulatory SASP factor which limits inflammaging at the expense of suppressing immune function.

\section{REFERENCES}

Abulizi, P., Loganathan, N., Zhao, D., Mele, T., Zhang, Y., Zwiep, T., et al. (2017). Growth Differentiation Factor-15 Deficiency Augments Inflammatory Response and Exacerbates Septic Heart and Renal Injury Induced by Lipopolysaccharide. Sci. Rep. 7, 1037. doi:10.1038/s41598017-00902-5

Adela, R., and Banerjee, S. K. (2015). GDF-15 as a Target and Biomarker for Diabetes and Cardiovascular Diseases: A Translational Prospective. J. Diabetes Res. 2015, 1-14. doi:10.1155/2015/490842

Aguilar-Recarte, D., Barroso, E., Gumà, A., Pizarro-Delgado, J., Peña, L., Ruart, M., et al. (2021). GDF15 Mediates the Metabolic Effects of PPAR $\beta / \delta$ by Activating AMPK. Cel Rep. 36, 109501. doi:10.1016/J.CELREP.2021.109501

Artz, A., Butz, S., and Vestweber, D. (2016). GDF-15 Inhibits Integrin Activation and Mouse Neutrophil Recruitment through the ALK-5/tgf-Brii Heterodimer. Blood 128, 529-541. doi:10.1182/blood-2016-01-696617

\section{DISCUSSION AND CONCLUSION}

Aging is associated with a substantial dysregulation of the immune system. GDF-15 is a stress-induced cytokine which is secreted under pro-inflammatory conditions and serves to limit inflammatory activation in many immune cell types. This suggests a potential inflammation-immunosuppression axis driven by GDF-15, whereby the protein is secreted during an inflammatory or immune activation event, and serves as a signal to assist in self-limiting or resolving the initial pro-inflammatory response.

By extension, chronic inflammation as induced by aging or senescence may therefore lead to chronic elevation of GDF-15 levels, leading to sustained suppression of the immune system. Recent evidence indicates that GDF-15 is highly associated with aging and increases across the lifespan, therefore GDF-15 is a strong potential link between inflammaging and immunosenescence. While there is some limited evidence suggesting that GDF-15 regulates immune function during aging, a great deal more work is necessary to convincingly demonstrate this. Nevertheless, GDF-15 is clearly an extremely important immunoregulatory cytokine which also has strong associations with biological aging. As such, further research in this area is likely to uncover additional links between GDF-15 and age-related immune dysfunction.

\section{AUTHOR CONTRIBUTIONS}

BP developed the topic, performed the literature review, and wrote the paper.

\section{FUNDING}

This research was partially supported by American Heart Association grants 18AIREA33960189 and 19TPA34910232 and a University of Memphis College of Health Sciences faculty research grant to $\mathrm{BP}$.

Baek, S. J., Kim, K.-S., Nixon, J. B., Wilson, L. C., and Eling, T. E. (2001) Cyclooxygenase Inhibitors Regulate the Expression of a TGF- $\beta$ Superfamily Member that Has Proapoptotic and Antitumorigenic Activities. Mol. Pharmacol. 59, 901-908. doi:10.1124/mol.59.4.901

Basisty, N., Kale, A., Jeon, O. H., Kuehnemann, C., Payne, T., Rao, C., et al. (2020). A Proteomic Atlas of Senescence-Associated Secretomes for Aging Biomarker Development. Plos Biol. 18, e3000599. doi:10.1371/journal.pbio.3000599

Bootcov, M. R., Bauskin, A. R., Valenzuela, S. M., Moore, A. G., Bansal, M., He, X. Y., et al. (1997). MIC-1, a Novel Macrophage Inhibitory Cytokine, Is a Divergent Member of the TGF- Superfamily. Proc. Natl. Acad. Sci. 94, 11514-11519. doi:10.1073/pnas.94.21.11514

Böttner, M., Laaff, M., Schechinger, B., Rappold, G., Unsicker, K., and SuterCrazzolara, C. (1999). Characterization of the Rat, Mouse, and Human Genes of Growth/differentiation Factor-15/macrophage Inhibiting Cytokine-1 (GDF15/MIC-1). Gene 237, 105-111. doi:10.1016/S0378-1119(99)00309-1

Buendgens, L., Yagmur, E., Bruensing, J., Herbers, U., Baeck, C., Trautwein, C., et al. (2017). Growth Differentiation Factor-15 Is a Predictor of Mortality in 
Critically Ill Patients with Sepsis. Dis. Markers 2017, 1-10. doi:10.1155/2017/ 5271203

Bułdak, Ł., Łabuzek, K., Bułdak, R. J., Kozłowski, M., MacHnik, G., Liber, S., et al. (2014). Metformin Affects Macrophages' Phenotype and Improves the Activity of Glutathione Peroxidase, Superoxide Dismutase, Catalase and Decreases Malondialdehyde Concentration in a Partially AMPK-independent Manner in LPS-Stimulated Human Monocytes/macrophages. Pharmacol. Rep. 66, 418-429. doi:10.1016/j.pharep.2013.11.008

Campisi, J. (2013). Aging, Cellular Senescence, and Cancer. Annu. Rev. Physiol. 75, 685-705. doi:10.1146/annurev-physiol-030212-183653

Campisi, J., Andersen, J. K., Kapahi, P., and Melov, S. (2011). Cellular Senescence: A Link between Cancer and Age-Related Degenerative Disease? Semin. Cancer Biol. 21, 354-359. doi:10.1016/j.semcancer.2011.09.001

Chen, Y. J., Liao, Y. J., Tram, V. T. N., Lin, C. H., Liao, K. C., and Liu, C. L. (2020). Alterations of Specific Lymphocytic Subsets with Aging and Age-Related Metabolic and Cardiovascular Diseases. Life 10, 246. doi:10.3390/ LIFE10100246

Conte, M., Martucci, M., Mosconi, G., Chiariello, A., Cappuccilli, M., Totti, V., et al. (2020). GDF15 Plasma Level Is Inversely Associated with Level of Physical Activity and Correlates with Markers of Inflammation and Muscle Weakness. Front. Immunol. 11, 915. doi:10.3389/FIMMU.2020.00915/BIBTEX

Cory, T. J., Emmons, R. S., Yarbro, J. R., Davis, K. L., and Pence, B. D. (2021). Metformin Suppresses Monocyte Immunometabolic Activation by SARS-CoV2 Spike Protein Subunit 1. Front. Immunol. 12, 733921. doi:10.3389/FIMMU. 2021.733921

Day, E. A., Ford, R. J., Smith, B. K., Mohammadi-Shemirani, P., Morrow, M. R., Gutgesell, R. M., et al. (2019). Metformin-induced Increases in GDF15 Are Important for Suppressing Appetite and Promoting Weight Loss. Nat. Metab. 1 (1), 1202-1208. doi:10.1038/s42255-019-0146-4

Doerstling, S., Hedberg, P., Öhrvik, J., Leppert, J., and Henriksen, E. (2018). Growth Differentiation Factor 15 in a Community-Based Sample: Agedependent Reference Limits and Prognostic Impact. Upsala J. Med. Sci. 123, 86-93. doi:10.1080/03009734.2018.1460427

Egerman, M. A., Cadena, S. M., Gilbert, J. A., Meyer, A., Nelson, H. N., Swalley, S. E., et al. (2015). GDF11 Increases with Age and Inhibits Skeletal Muscle Regeneration. Cel Metab. 22, 164-174. doi:10.1016/j.cmet.2015.05.010

Emmerson, P. J., Wang, F., Du, Y., Liu, Q., Pickard, R. T., Gonciarz, M. D., et al. (2017). The Metabolic Effects of GDF15 Are Mediated by the Orphan Receptor GFRAL. Nat. Med. 23, 1215-1219. doi:10.1038/nm.4393

Esalatmanesh, K., Fayyazi, H., Esalatmanesh, R., and Khabbazi, A. (2020). The Association between Serum Levels of Growth Differentiation Factor-15 and Rheumatoid Arthritis Activity. Int. J. Clin. Pract. 74, e13564. doi:10.1111/IJCP. 13564

Franceschi, C., and Campisi, J. (2014). Chronic Inflammation (Inflammaging) and its Potential Contribution to Age-Associated Diseases. J. Gerontol. Ser. A: Biol. Sci. Med. Sci. 69, S4-S9. doi:10.1093/gerona/glu057

Freund, A., Orjalo, A. V., Desprez, P.-Y., and Campisi, J. (2010). Inflammatory Networks during Cellular Senescence: Causes and Consequences. Trends Mol. Med. 16, 238-246. doi:10.1016/j.molmed.2010.03.003

Fuchs, T., Trollor, J. N., Crawford, J., Brown, D. A., Baune, B. T., Samaras, K., et al. (2013). Macrophage Inhibitory Cytokine-1 Is Associated with Cognitive Impairment and Predicts Cognitive Decline - the Sydney Memory and Aging Study. Aging Cell 12, 882-889. doi:10.1111/acel.12116

Fulop, T., Larbi, A., Dupuis, G., Le Page, A., Frost, E. H., Cohen, A. A., et al. (2018). Immunosenescence and Inflamm-Aging as Two Sides of the Same coin: Friends or Foes? Front. Immunol. 8, 1960. doi:10.3389/fimmu.2017.01960

Furman, D., Campisi, J., Verdin, E., Carrera-Bastos, P., Targ, S., Franceschi, C., et al. (2019). Chronic Inflammation in the Etiology of Disease across the Life Span. Nat. Med. 25, 1822-1832. doi:10.1038/s41591-019-0675-0

Goronzy, J. J., and Weyand, C. M. (2013). Understanding Immunosenescence to Improve Responses to Vaccines. Nat. Immunol. 14, 428-436. doi:10.1038/ni. 2588

Hearps, A. C., Martin, G. E., Angelovich, T. A., Cheng, W.-J., Maisa, A., Landay, A. L., et al. (2012). Aging Is Associated with Chronic Innate Immune Activation and Dysregulation of Monocyte Phenotype and Function. Aging Cell 11, 867-875. doi:10.1111/j.1474-9726.2012.00851.x

Heduschke, A., Ackermann, K., Wilhelm, B., Mey, L., Bonaterra, G. A., Kinscherf, R., et al. (2021). GDF-15 Deficiency Reduces Autophagic Activity in Human
Macrophages In Vitro and Decreases P62-Accumulation in Atherosclerotic Lesions in Mice. Cells 10, 2346. doi:10.3390/CELLS10092346

Ho, J. E., Mahajan, A., Chen, M.-H., Larson, M. G., McCabe, E. L., Ghorbani, A., et al. (2012). Clinical and Genetic Correlates of Growth Differentiation Factor 15 in the Community. Clin. Chem. 58, 1582-1591. doi:10.1373/clinchem.2012. 190322

Hromas, R., Hufford, M., Sutton, J., Xu, D., Li, Y., and Lu, L. (1997). PLAB, a Novel Placental Bone Morphogenetic Protein. Biochim. Biophys. Acta (Bba) - Gene Struct. Expr. 1354, 40-44. doi:10.1016/S0167-4781(97)00122-X

Jamaiyar, A., Wan, W., Janota, D. M., Enrick, M. K., Chilian, W. M., and Yin, L. (2017). The Versatility and Paradox of GDF 11. Pharmacol. Ther. 175, 28-34. doi:10.1016/j.pharmthera.2017.02.032

Jung, S.-B., Choi, M. J., Ryu, D., Yi, H.-S., Lee, S. E., Chang, J. Y., et al. (2018). Reduced Oxidative Capacity in Macrophages Results in Systemic Insulin Resistance. Nat. Commun. 9, 1551. doi:10.1038/s41467-018-03998-z

Kapetanovic, R., Bokil, N. J., and Sweet, M. J. (2015). Innate Immune Perturbations, Accumulating DAMPs and Inflammasome Dysregulation: A Ticking Time Bomb in Ageing. Ageing Res. Rev. 24, 40-53. doi:10.1016/j.arr.2015.02.005

Katsimpardi, L., Litterman, N. K., Schein, P. A., Miller, C. M., Loffredo, F. S., Wojtkiewicz, G. R., et al. (2014). Vascular and Neurogenic Rejuvenation of the Aging Mouse Brain by Young Systemic Factors. Science 344, 630-634. doi:10. 1126/science.1251141

Kempf, T., Zarbock, A., Widera, C., Butz, S., Stadtmann, A., Rossaint, J., et al. (2011). GDF-15 Is an Inhibitor of Leukocyte Integrin Activation Required for Survival after Myocardial Infarction in Mice. Nat. Med. 17, 581-588. doi:10. 1038/nm.2354

Kim, J., Kwak, H. J., Cha, J.-Y., Jeong, Y.-S., Rhee, S. D., Kim, K. R., et al. (2014). Metformin Suppresses Lipopolysaccharide (LPS)-induced Inflammatory Response in Murine Macrophages via Activating Transcription Factor-3 (ATF-3) Induction. J. Biol. Chem. 289, 23246-23255. doi:10.1074/jbc.M114. 577908

Kim, J. M., Kosak, J. P., Kim, J. K., Kissling, G., Germolec, D. R., Zeldin, D. C., et al. (2013). NAG-1/GDF15 Transgenic Mouse Has Less White Adipose Tissue and a Reduced Inflammatory Response. Mediators Inflamm. 2013, 1-10. doi:10. $1155 / 2013 / 641851$

Kleinertz, H., Hepner-Schefczyk, M., Ehnert, S., Claus, M., Halbgebauer, R., Boller, L., et al. (2019). Circulating Growth/differentiation Factor 15 Is Associated with Human CD56bright Natural Killer Cell Dysfunction and Nosocomial Infection in Severe Systemic Inflammation. EBioMedicine 43, 380-391. doi:10.1016/j. ebiom.2019.04.018

Lawton, L. N., Bonaldo, M. D. F., Jelenc, P. C., Qiu, L., Baumes, S. A., Marcelino, R. A., et al. (1997). Identification of a Novel Member of the TGF-Beta Superfamily Highly Expressed in Human Placenta. Gene 203, 17-26. doi:10.1016/S03781119(97)00485-X

Lee, S. E., Kang, S. G., Choi, M. J., Jung, S.-B., Ryu, M. J., Chung, H. K., et al. (2017). Growth Differentiation Factor 15 Mediates Systemic Glucose Regulatory Action of T-Helper Type 2 Cytokines. Diabetes 66, 2774-2788. doi:10.2337/ $\mathrm{db} 17-0333$

Linton, P.-J., and Thoman, M. L. (2014). Immunosenescence in Monocytes, Macrophages, and Dendritic Cells: Lessons Learned from the Lung and Heart. Immunol. Lett. 162, 290-297. doi:10.1016/j.imlet.2014.06.017

Lodi, R. S., Yu, B., Xia, L., and Liu, F. (2021). Roles and Regulation of Growth Differentiation Factor-15 in the Immune and Tumor Microenvironment. Hum. Immunol. 82, 937-944. doi:10.1016/J.HUMIMM.2021.06.007

Loffredo, F. S., Steinhauser, M. L., Jay, S. M., Gannon, J., Pancoast, J. R., Yalamanchi, P., et al. (2013). Growth Differentiation Factor 11 Is a Circulating Factor that Reverses Age-Related Cardiac Hypertrophy. Cell 153, 828-839. doi:10.1016/j.cell.2013.04.015

López-Otín, C., Blasco, M. A., Partridge, L., Serrano, M., and Kroemer, G. (2013). The Hallmarks of Aging. Cell 153, 1194-1217. doi:10.1016/j.cell.2013.05.039

Luan, H. H., Wang, A., Hilliard, B. K., Carvalho, F., Rosen, C. E., Ahasic, A. M., et al. (2019). GDF15 Is an Inflammation-Induced Central Mediator of Tissue Tolerance. Cell 178, 1231-1244. doi:10.1016/j.cell.2019.07.033

Montgomery, R. R., and Shaw, A. C. (2015). Paradoxical Changes in Innate Immunity in Aging: Recent Progress and New Directions. J. Leukoc. Biol. 98, 937-943. doi:10.1189/jlb.5mr0315-104r

Moon, J. S., Goeminne, L. J. E., Kim, J. T., Tian, J. W., Kim, S. H., Nga, H. T., et al. (2020). Growth Differentiation Factor 15 Protects against the Aging-mediated 
Systemic Inflammatory Response in Humans and Mice. Aging Cell 19, e13195. doi:10.1111/acel.13195

Mullican, S. E., Lin-Schmidt, X., Chin, C.-N., Chavez, J. A., Furman, J. L., Armstrong, A. A., et al. (2017). GFRAL Is the Receptor for GDF15 and the Ligand Promotes Weight Loss in Mice and Nonhuman Primates. Nat. Med. 23, 1150-1157. doi:10.1038/nm.4392

Niccoli, T., and Partridge, L. (2012). Ageing as a Risk Factor for Disease. Curr. Biol. 22, R741-R752. doi:10.1016/j.cub.2012.07.024

Notz, Q., Schmalzing, M., Wedekink, F., Schlesinger, T., Gernert, M., Herrmann, J., et al. (2020). Pro- and Anti-inflammatory Responses in Severe COVID-19Induced Acute Respiratory Distress Syndrome-An Observational Pilot Study. Front. Immunol. 11, 581338. doi:10.3389/FIMMU.2020.581338/FULL

Pence, B. D., and Yarbro, J. R. (2018). Aging Impairs Mitochondrial Respiratory Capacity in Classical Monocytes. Exp. Gerontol. 108, 112-117. doi:10.1016/j. exger.2018.04.008

Pence, B. D., and Yarbro, J. R. (2019). Classical Monocytes Maintain Ex Vivo Glycolytic Metabolism and Early but Not Later Inflammatory Responses in Older Adults. Immun. Ageing 16, 3. doi:10.1186/s12979-019-0143-1

Pence, B. D., Yarbro, J. R., and Emmons, R. S. (2021). Growth Differentiation Factor-15 Is Associated with Age-related Monocyte Dysfunction. Aging Med. 4, 47-52. doi:10.1002/agm2.12128

Poggioli, T., Vujic, A., Yang, P., MacIas-Trevino, C., Uygur, A., Loffredo, F. S., et al. (2016). Circulating Growth Differentiation Factor 11/8 Levels Decline with Age. Circ. Res. 118, 29-37. doi:10.1161/CIRCRESAHA.115.307521

Preusch, M. R., Baeuerle, M., Albrecht, C., Blessing, E., Bischof, M., Katus, H. A., et al. (2013). GDF-15 Protects from Macrophage Accumulation in a Mousemodel of Advanced Atherosclerosis. Eur. J. Med. Res. 18, 19. doi:10. 1186/2047-783X-18-19

Qing, L., Fu, J., Wu, P., Zhou, Z., Yu, F., and Tang, J. (2019). Metformin Induces the M2 Macrophage Polarization to Accelerate the Wound Healing via Regulating AMPK/mTOR/NLRP3 Inflammasome Singling Pathway. Am. J. Transl. Res. 11, 655-668.

Ratnam, N. M., Peterson, J. M., Talbert, E. E., Ladner, K. J., Rajasekera, P. V., Schmidt, C. R., et al. (2017). NF-кB Regulates GDF-15 to Suppress Macrophage Surveillance during Early Tumor Development. J. Clin. Invest. 127, 3796-3809. doi:10.1172/JCI91561

Renshaw, M., Rockwell, J., Engleman, C., Gewirtz, A., Katz, J., and Sambhara, S. (2002). Cutting Edge: Impaired Toll-like Receptor Expression and Function in Aging. J. Immunol. 169, 4697-4701. doi:10.4049/jimmunol. 169.9.4697

Rochette, L., Zeller, M., Cottin, Y., and Vergely, C. (2021). GDF15: an Emerging Modulator of Immunity and a Strategy in COVID-19 in Association with Iron Metabolism. Trends Endocrinol. Metab. 32, 875-889. doi:10.1016/J.TEM.2021. 08.011

Roth, P., Junker, M., Tritschler, I., Mittelbronn, M., Dombrowski, Y., Breit, S. N., et al. (2010). GDF-15 Contributes to Proliferation and Immune Escape of Malignant Gliomas. Clin. Cancer Res. 16, 3851-3859. doi:10.1158/1078-0432. CCR-10-0705

Sayed, N., Huang, Y., Nguyen, K., Krejciova-Rajaniemi, Z., Grawe, A. P., Gao, T., et al. (2021). An Inflammatory Aging Clock (iAge) Based on Deep Learning Tracks Multimorbidity, Immunosenescence, Frailty and Cardiovascular Aging. Nat. Aging 1 (1), 598-615. doi:10.1038/s43587-021-00082-y

Segerer, S. E., Rieger, L., Kapp, M., Dombrowski, Y., Müller, N., Dietl, J., et al. (2012). MIC-1 (A Multifunctional Modulator of Dendritic Cell Phenotype and Function) Is Produced by Decidual Stromal Cells and Trophoblasts. Hum. Reprod. 27, 200-209. doi:10.1093/humrep/der358

Sinha, M., Jang, Y. C., Oh, J., Khong, D., Wu, E. Y., Manohar, R., et al. (2014). Restoring Systemic GDF11 Levels Reverses Age-Related Dysfunction in Mouse Skeletal Muscle. Science 344, 649-652. doi:10.1126/science.1251152

Smith, S. C., Zhang, X., Zhang, X., Gross, P., Starosta, T., Mohsin, S., et al. (2015). GDF11 Does Not Rescue Aging-Related Pathological Hypertrophy. Circ. Res. 117, 926-932. doi:10.1161/CIRCRESAHA.115.307527

Soberanes, S., Misharin, A. V., Jairaman, A., Morales-Nebreda, L., McQuattiePimentel, A. C., Cho, T., et al. (2019). Metformin Targets Mitochondrial Electron Transport to Reduce Air-Pollution-Induced Thrombosis. Cel Metab. 29, 335-347.e5. doi:10.1016/j.cmet.2018.09.019

Tan, M., Wang, Y., Guan, K., and Sun, Y. (2000). PTGF-beta , a Type Beta Transforming Growth Factor (TGF-Beta) Superfamily Member, Is a P53
Target Gene that Inhibits Tumor Cell Growth via TGF-Beta Signaling Pathway. Proc. Natl. Acad. Sci. 97, 109-114. doi:10.1073/pnas.97.1.109

Tanaka, T., Basisty, N., Fantoni, G., Candia, J., Moore, A. Z., Biancotto, A., et al. (2020). Plasma Proteomic Biomarker Signature of Age Predicts Health and Life Span. Elife 9, 1-24. doi:10.7554/ELIFE.61073

Tanaka, T., Biancotto, A., Moaddel, R., Moore, A. Z., Gonzalez-Freire, M., Aon, M. A., et al. (2018). Plasma Proteomic Signature of Age in Healthy Humans. Aging Cell 17, e12799. doi:10.1111/acel.12799

Tavenier, J., Rasmussen, L. J. H., Andersen, A. L., Houlind, M. B., Langkilde, A., Andersen, O., et al. (2021). Association of GDF15 with Inflammation and Physical Function during Aging and Recovery after Acute Hospitalization: A Longitudinal Study of Older Patients and Age-Matched Controls. J. Gerontol. A. Biol. Sci. Med. Sci. 76, 964-974. doi:10.1093/GERONA/GLAB011

Unsicker, K., Spittau, B., and Krieglstein, K. (2013). The Multiple Facets of the TGF- $\beta$ Family Cytokine Growth/differentiation Factor-15/macrophage Inhibitory Cytokine-1. Cytokine Growth Factor. Rev. 24, 373-384. doi:10. 1016/j.cytogfr.2013.05.003

Wang, X., Chrysovergis, K., Kosak, J., and Eling, T. E. (2014). Lower NLRP3 Inflammasome Activity in NAG-1 Transgenic Mice Is Linked to a Resistance to Obesity and Increased Insulin Sensitivity. Obesity 22, 1256-1263. doi:10.1002/ oby. 20638

Wiklund, F. E., Bennet, A. M., Magnusson, P. K. E., Eriksson, U. K., Lindmark, F., $\mathrm{Wu}$, L., et al. (2010). Macrophage Inhibitory Cytokine-1 (MIC-1/GDF15): A New Marker of All-Cause Mortality. Aging Cell 9, 1057-1064. doi:10.1111/j. 1474-9726.2010.00629.x

Wischhusen, J., Melero, I., and Fridman, W. H. (2020). Growth/Differentiation Factor-15 (GDF-15): From Biomarker to Novel Targetable Immune Checkpoint. Front. Immunol. 11, 951. doi:10.3389/FIMMU.2020.00951

Wollert, K. C., Kempf, T., and Wallentin, L. (2017). Growth Differentiation Factor 15 as a Biomarker in Cardiovascular Disease. Clin. Chem. 63, 140-151. doi:10. 1373/clinchem.2016.255174

Wong, C., and Goldstein, D. R. (2013). Impact of Aging on Antigen Presentation Cell Function of Dendritic Cells. Curr. Opin. Immunol. 25, 535-541. doi:10. 1016/J.COI.2013.05.016

Wu, Q., Jiang, D., Schaefer, N. R., Harmacek, L., O'Connor, B. P., Eling, T. E., et al. (2018). Overproduction of Growth Differentiation Factor 15 Promotes Human Rhinovirus Infection and Virus-Induced Inflammation in the Lung. Am. J. Physiol Lung Cell Mol. Physiol. 314, L514-L527. doi:10.1152/ajplung. 00324.2017

Xian, H., Liu, Y., Rundberg Nilsson, A., Gatchalian, R., Crother, T. R., Tourtellotte, W. G., et al. (2021). Metformin Inhibition of Mitochondrial ATP and DNA Synthesis Abrogates NLRP3 Inflammasome Activation and Pulmonary Inflammation. Immunity 54, 1463-1477.e11. doi:10.1016/j.immuni.2021. 05.004

Yamaguchi, Y., Zampino, M., Tanaka, T., Bandinelli, S., Osawa, Y., Ferrucci, L., et al. (2021). Elevated Plasma Growth and Differentiation Factor 15 Predicts Incident Anemia in Older Adults Aged 60 Years and Older. J. Gerontol. Ser. A. 76, 1192-1197. doi:10.1093/GERONA/GLAA324

Yang, L., Chang, C.-C., Sun, Z., Madsen, D., Zhu, H., Padkjær, S. B., et al. (2017). GFRAL Is the Receptor for GDF15 and Is Required for the Anti-obesity Effects of the Ligand. Nat. Med. 23, 1158-1166. doi:10.1038/nm.4394

Yarbro, J. R., Emmons, R. S., and Pence, B. D. (2020). Macrophage Immunometabolism and Inflammaging: Roles of Mitochondrial Dysfunction, Cellular Senescence, CD38, and NAD. Immunometabolism 2, e200026. doi:10.20900/immunometab20200026

Yatsuga, S., Fujita, Y., Ishii, A., Fukumoto, Y., Arahata, H., Kakuma, T., et al. (2015). Growth Differentiation Factor 15 as a Useful Biomarker for Mitochondrial Disorders. Ann. Neurol. 78, 814-823. doi:10.1002/ana. 24506

Yokoyama-Kobayashi, M., Saeki, M., Sekine, S., and Kato, S. (1997). Human cDNA Encoding a Novel TGF- Superfamily Protein Highly Expressed in Placenta. J. Biochem. 122, 622-626. doi:10.1093/oxfordjournals.jbchem. a021798

Zhang, M., Pan, K., Liu, Q., Zhou, X., Jiang, T., and Li, Y. (2016). Growth Differentiation Factor 15 May Protect the Myocardium from No-Reflow by Inhibiting the Inflammatory-like Response that Predominantly Involves Neutrophil Infiltration. Mol. Med. Rep. 13, 623-632. doi:10.3892/mmr.2015. 4573 
Zhang, Y., Zhang, G., Liu, Y., Chen, R., Zhao, D., McAlister, V., et al. (2018). GDF15 Regulates Malat-1 Circular RNA and Inactivates NFkB Signaling Leading to Immune Tolerogenic DCs for Preventing Alloimmune Rejection in Heart Transplantation. Front. Immunol. 9, 2407. doi:10.3389/fimmu.2018. 02407

Zhao, B., Li, H., Cao, S., Zhong, W., Li, B., Jia, W., et al. (2021). Negative Regulators of Inflammation Response to the Dynamic Expression of Cytokines in DF-1 and MDCK Cells Infected by Avian Influenza Viruses. Inflammation. doi:10. 1007/S10753-021-01568-Y

Zhou, D., Borsa, M., and Simon, A. K. (2021). Hallmarks and Detection Techniques of Cellular Senescence and Cellular Ageing in Immune Cells. Aging Cell 20, e13316. doi:10.1111/ACEL.13316

Zhou, Z., Li, W., Song, Y., Wang, L., Zhang, K., Yang, J., et al. (2013). Growth Differentiation Factor-15 Suppresses Maturation and Function of Dendritic Cells and Inhibits Tumor-specific Immune Response. PLoS One 8, e78618. doi:10.1371/journal.pone.0078618
Conflict of Interest: The author declares that the research was conducted in the absence of any commercial or financial relationships that could be construed as a potential conflict of interest.

Publisher's Note: All claims expressed in this article are solely those of the authors and do not necessarily represent those of their affiliated organizations, or those of the publisher, the editors and the reviewers. Any product that may be evaluated in this article, or claim that may be made by its manufacturer, is not guaranteed or endorsed by the publisher.

Copyright (C) 2022 Pence. This is an open-access article distributed under the terms of the Creative Commons Attribution License (CC BY). The use, distribution or reproduction in other forums is permitted, provided the original author(s) and the copyright owner(s) are credited and that the original publication in this journal is cited, in accordance with accepted academic practice. No use, distribution or reproduction is permitted which does not comply with these terms. 